\title{
Metamaterial/graphene amplitude and frequency modulators for the active control of terahertz quantum cascade lasers
}

\author{
R. Degl’Innocenti ${ }^{* a}$, Stephen J. Kindness ${ }^{\mathrm{b}}$, Nikita W. Almond ${ }^{\mathrm{b}}$, Binbin Wei ${ }^{\mathrm{b}}$, Robert Wallis ${ }^{\mathrm{b}}$, Varun \\ S. Kamboj ${ }^{\mathrm{b}}$, Philipp Braeuninger-Weimer ${ }^{\mathrm{c}}$, Stephan Hofmann ${ }^{\mathrm{c}}$, Harvey E. Beere ${ }^{\mathrm{b}}$, David A. Ritchie ${ }^{\mathrm{b}}$ \\ ${ }^{\mathrm{a}}$ Department of Engineering, Lancaster University, Lancaster, LA1 4YW, UK; ${ }^{\mathrm{b}}$ Cavendish \\ Laboratory, University of Cambridge, J. J. Thomson Avenue, Cambridge, CB3 0HE, UK; \\ ${ }^{\mathrm{c}}$ Department of Engineering, University of Cambridge, $9 \mathrm{~J}$. J. Thomson Avenue, Cambridge, CB3 \\ OFA, UK.
}

\begin{abstract}
Hybrid metamaterial/graphene amplitude and frequency modulators have been implemented as external optoelectronic mirrors in external cavity configurations with terahertz quantum cascade lasers (QCLs). These devices' tunability is accomplished via the interplay between metamaterial resonant units, normally engineered in mm-size arrays, and graphene. The integration of these devices in external cavity QCLs offers unique emission features and realizes an unprecedented studied regime. The implementation of an external amplitude modulation allows the full switching of laser emission in single mode operation by electrostatically gating graphene. The introduction of more dispersive tunable architectures in frequency modulators yields additionally an all-electronic spectral laser bistability.
\end{abstract}

Keywords: Metamaterial, amplitude modulator, frequency modulator, quantum cascade lasers, graphene

\section{INTRODUCTION}

Fast, integrated optoelectronic devices, such as amplitude and frequency modulators, are in high demand in terahertz (THz) science and technology ${ }^{1}$ as they bridge the gap between sources, such as the quantum cascade laser (QCL) ${ }^{2}$, and broadband fs-pulse based time domain spectroscopic systems (THz-TDS) ${ }^{3}$, and detectors, e. g., ${ }^{4,5}$, thus enabling a myriad of applications in spectroscopy, imaging and communications 6 . In fact, for the full exploitation of these applications it is necessary to achieve fast, active control of the $\mathrm{THz}$ radiation properties, including frequency, amplitude, phase and polarization. THz QCLs are widely used solid-state sources. Because of their unique characteristics, such as narrow linewidth, high-stability and Watt-level power ${ }^{7}$, these devices find applications in several fields such as solid-state and gas spectroscopy ${ }^{8}$ e. g. for monitoring the presence/concentration of noxious or greenhouse gases with high precision., or in astronomy as local oscillators ${ }^{9}$, and imaging ${ }^{10}$. The achievement of an external and efficient active control of QCL emission, would hence have a great impact in all the aforementioned applications and would help to enlarge the application horizon for this source. The proposed approach combines amplitude/frequency modulators used as fastreconfigurable mirrors in an external cavity configuration with quantum cascade amplifiers (QCA) ${ }^{11}$. The difficulties in realizing $\mathrm{THz}$ tunable devices derives from the fact that this frequency range lies between the photonic and electronic range. Micromechanical systems (MEMS) although having remarkable features in terms of modulation depth, fail to address a few issues which are fundamental for QCL modulation, such as modulation speed and versatility ${ }^{1}$. Metal plasmonic/metamaterial-based devices provide a unique, versatile solution because of their lithographically defined artificial optical resonances which are subwavelength in nature, leading to high optical field concentrations and providing high efficiency ${ }^{12}$. One common solution to achieve an active tuning of metamaterial-based devices consists in their integration with materials which have a variable conductivity, capable of damping or fundamentally changing the optical response, e.g. photoactive silicon, ${ }^{13}$ or graphene ${ }^{14}$. The conductivity of silicon can be modified by using an infrared source with photon energy above the band gap to excite charge carriers. However, this approach requires an additional optical set-up for active control, strongly limiting its applicability out of the laboratory environment. Graphene is an ideal material for modulation as it can be easily integrated into the metamaterial device fabrication process and possesses a large conductivity range which can be electrically tuned at high speeds ${ }^{15,16}$. Accordingly, amplitude and frequency modulators were designed based on the interplay between metallic metamaterials resonant units, typically

*r.deglinnocenti@lancaster.ac.uk; phone +44 (0)1524510139 
arranged in mm-size arrays, and chemical vapor deposition (CVD) grown graphene ${ }^{17}$, providing the active component. Amplitude modulator architectures were realized by fabricating metallic split-ring resonator arrays on top of a large graphene patch transferred onto $\mathrm{SiO}_{2} / \mathrm{Si}$ substrates. The engineering of the inherently more challenging frequency tunable devices was based on a more complex, coupled resonator design interacting with patterned graphene, which produces optical features reminiscent of electromagnetically induced transparency (EIT). EIT traditionally refers to quantum destructive interference between excitation states in atomic systems, however, this effect has been shown to have classical analogs using coupled bright and dark plasmonic resonators ${ }^{18}$. Such metamaterial structures are of interest as EIT produces an extreme modification of the dispersion properties, which can then be used in different experimental configurations. The achievement of an active frequency control is of fundamental importance in a variety of scenarios. A continuous frequency tuning over a range larger than $10 \mathrm{MHz}$ has proved to be a difficult task and the few successful approaches which have accomplished this goal, required either a non-trivial laser arrangement ${ }^{19}$, or a complex engineering of the photonic emission ${ }^{20}$ and are typically based on a mechanical or thermal tuning which limit their versatility and affect their reconfiguration speed. The combination of more dispersive elements such as frequency modulators with amplitude modulators, would also achieve a fundamental step towards the realization a more complex optoelectronic device with reconfigurable dispersive capabilities. Such a device can be implemented, e.g., for the development of fs-pulse mode locked QCLs. The achievement of sub-ps pulses still remains elusive, despite great progresses have been reported recently ${ }^{21}$. An alternative and innovative approach would consist in the design of a flexible and tunable optoelectronic external mirror capable of modulating the amplitude and phase of the light fed back into the laser cavity and of compensating the group delay dispersion over a $1 \mathrm{THz}$ bandwidth. The integrated amplitude modulators have shown themselves capable of providing a fast, electrical modulation and, in combination with QCLs, $100 \%$ amplitude modulation depth ${ }^{22}$ and efficient active stabilization of these lasers ${ }^{23}$ have also been demonstrated. Importantly, by adjusting the QCL emission frequency to the operation frequency of the amplitude modulators, these devices have been efficient in reinforcing single mode operation, a fundamental pre-requisite in many fields, e.g. spectroscopy. Unique QCL emission features emerged when using more frequency dispersive optoelectronic mirrors in external cavity quantum cascade laser (EC-QCL) arrangements, such as controlled optical bistability, an all-electronic switch between two different frequencies emitted in single-mode, which can be used in frequency-shift-key in communication protocols.

\section{MODULATORS: DESIGNS AND SIMULATIONS}

\subsection{Operating principles of amplitude/frequency modulators}

The amplitude modulator experiments are based on split ring resonators (SRR) metamaterials. The SRRs are wellstudied resonant elements engineered as metallic (Ti/Au, 10/100 nm) features over a $\mathrm{SiO}_{2} / \mathrm{Si}(290 \mathrm{~nm} / 500 \mu \mathrm{m})$ substrate. Chemical vapour deposition (CVD) graphene has been transferred onto the $\mathrm{SiO}_{2} / \mathrm{Si}$ chip, before the fabrication of the arrays and bonding pads via electron beam lithography and metallic evaporation. The device is encapsulated with $80 \mathrm{~nm}$ $\mathrm{Al}_{2} \mathrm{O}_{3}$ dielectric layer via atomic layer deposition to reduce graphene hysteresis and the graphene unintentional doping, as well as to lower the voltage required to achieve the Dirac point. More details on fabrication are reported in reference ${ }^{22}$. The resonance targeted is the LC resonance, which is excited by the incoming radiation having a component of the Efield perpendicular to the central gap at the resonant frequency. In analogy with a circuit model the inductance is located in the metallic arms, the capacitance C of the SRR is defined by the gap, and graphene acts as variable resistance. The induced currents in the arms of the SRR, recombine across the capacitive gap where the maximum of the E-field is concentrated and strongly overlaps with graphene. By electrostatically changing the carrier concentration in the graphene layer it is possible to actively damp the resonance. A scanning electron microscope (SEM) top view picture of the SRR arrays is shown in Fig. 1(a); the graphene is covering the whole area. EIT frequency modulators have been realized with similar fabrication process, but graphene was transferred and patterned after the definition of the metamaterial resonant units and was left exposed. The unit cell of these devices is based on the interplay between two strongly coupled metamaterial features, having a similar resonant frequency, but interacting differently with the incoming radiation. A SEM picture of the basic EIT unit is showed in Fig. 1(b). The top C-shaped feature, called the "bright" resonator, is strongly coupled to the incident THz radiation having a large horizontal component parallel to the incoming E-field. The bottom "dark" resonator instead, is mainly excited, through the capacitive coupling with the bright resonator, because of the shorter length parallel to the incident field. The dark resonator presents a short gap shunted by the graphene patterned area as shown in Fig. 1(b). The strong coupling between these two resonators induces a splitting of the standard bare resonances into two hybridized modes, with a low frequency 'bonding' resonance and a high frequency 'anti-bonding' resonance. By electrostatically gating graphene in contact with the sub-radiant resonator, it is possible to tune the Q- 
factor of the dark element, and therefore the strength of the coupling with the bright resonator, whose resonance can be continuously tuned. The single units are arranged into arrays which are connected to the bonding pads via metallic wires. The SRR arrays have a total area of $3 \times 3 \mathrm{~mm}^{2}$ while the EIT devices measure $1.7 \times 1.7 \mathrm{~mm}^{2}$. The sample is then wire bonded for electrostatic gating of the graphene through the p-doped Si substrate, as shown in Fig. 1 (c).
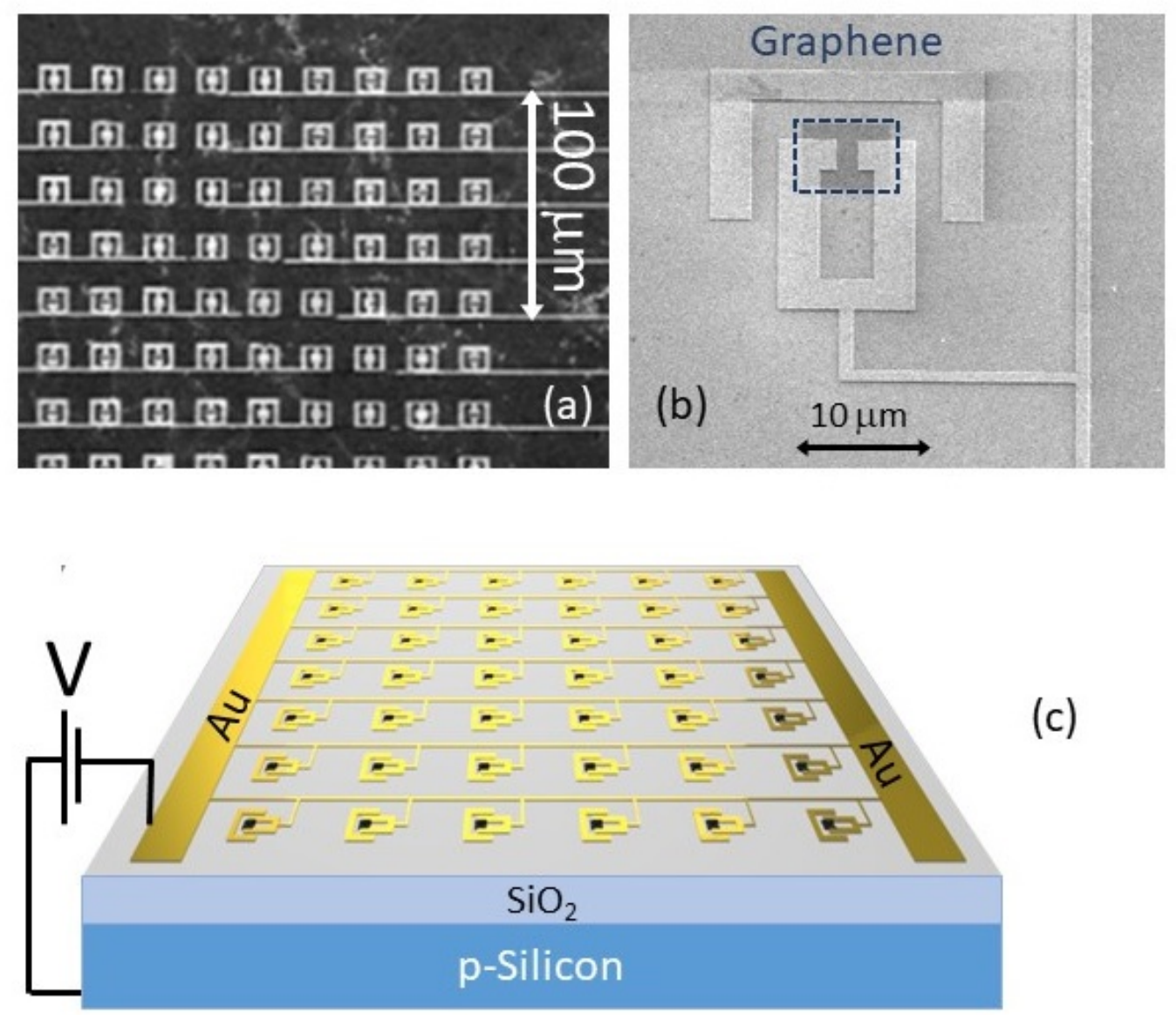

Figure 1. (a) SEM picture of the SRR arrays for the amplitude modulators. (b) SEM picture of a single resonant unit for the EIT device used for frequency modulation. The graphene patterned region, showed by the dashed area, short the gap in the dark resonators. (c) Schematic of the final device.

\subsection{Simulations}

The design of these devices necessarily passes through a simulation and modeling step performed with an electromagnetic finite element method (FEM) software; COMSOL Multiphysics, RF module. A single unit cell with Floquet periodic boundary conditions has been implemented in the simulations. The dielectric properties of the various materials, together with a Drude model used to model the graphene and the p-doped Si substrate, were inserted in the simulations and are reported in reference ${ }^{22}$. The incoming $\mathrm{THz}$ radiation is simulated by introducing a port above the SRR/EIT structure linearly polarized to excite the resonant elements. The reflection and transmission coefficients are acquired by monitoring and recording the $S_{11}$ and $S_{21}$ parameters respectively at different graphene conductivity levels. The graphene conductivity range considered in the simulations spans from $0.1 \mathrm{mS}$ to $1.6 \mathrm{mS}$, compatible with the conductivity range typically achieved with CVD grown graphene and consistent with the values reported in our previous experiments. Figure 2 shows the results of the simulations for both the SRR and the EIT devices. Figure 2(a)- the SRR unit was designed to be resonant at $2.85 \mathrm{THz}$, and as expected at resonance the electric-field is mostly concentrated in the capacitive gap. The red arrows show the current paths. From the conductivity sweeping, it is clear that the resonance is strongly dampened for high graphene carrier concentration, as expected, while the resonance dispersion is marginally affected. Figure 2(b) instead shows the metamaterial basic element targeting frequency modulators, which were designed 
to have a larger dispersion. The electric field of this complex resonant unit is presented for the bonding $\mathrm{f}_{1}$ and antibonding $\mathrm{f}_{2}$ frequencies at different conductivity levels, $0.1 \mathrm{~ms}$ and $1.1 \mathrm{mS}$ respectively. The bonding frequency presents highest E-field concentration at the end of the C-shaped feature, indirectly exciting the inner resonant feature, which has maximum E-field in the gap, where the graphene patch has been transferred. At low conductivity the two resonant elements having similar resonant frequencies are shifted apart, in analogy with the classical model of harmonic oscillators, because of their mutual interaction. As the graphene conductivity is increased, $\mathrm{f}_{1}$ is continuously blue shifted by $\sim 100 \mathrm{GHz}$ towards the resonance of the bare bright resonator with only a minor impact on the $\mathrm{Q}$ factor. At the same time, the $\mathrm{Q}$ factor of the antibonding frequency $\mathrm{f}_{2}$ is quickly degraded as the graphene conductivity increases and the strongly dampened dark resonator no longer has an influence on the bright resonator.
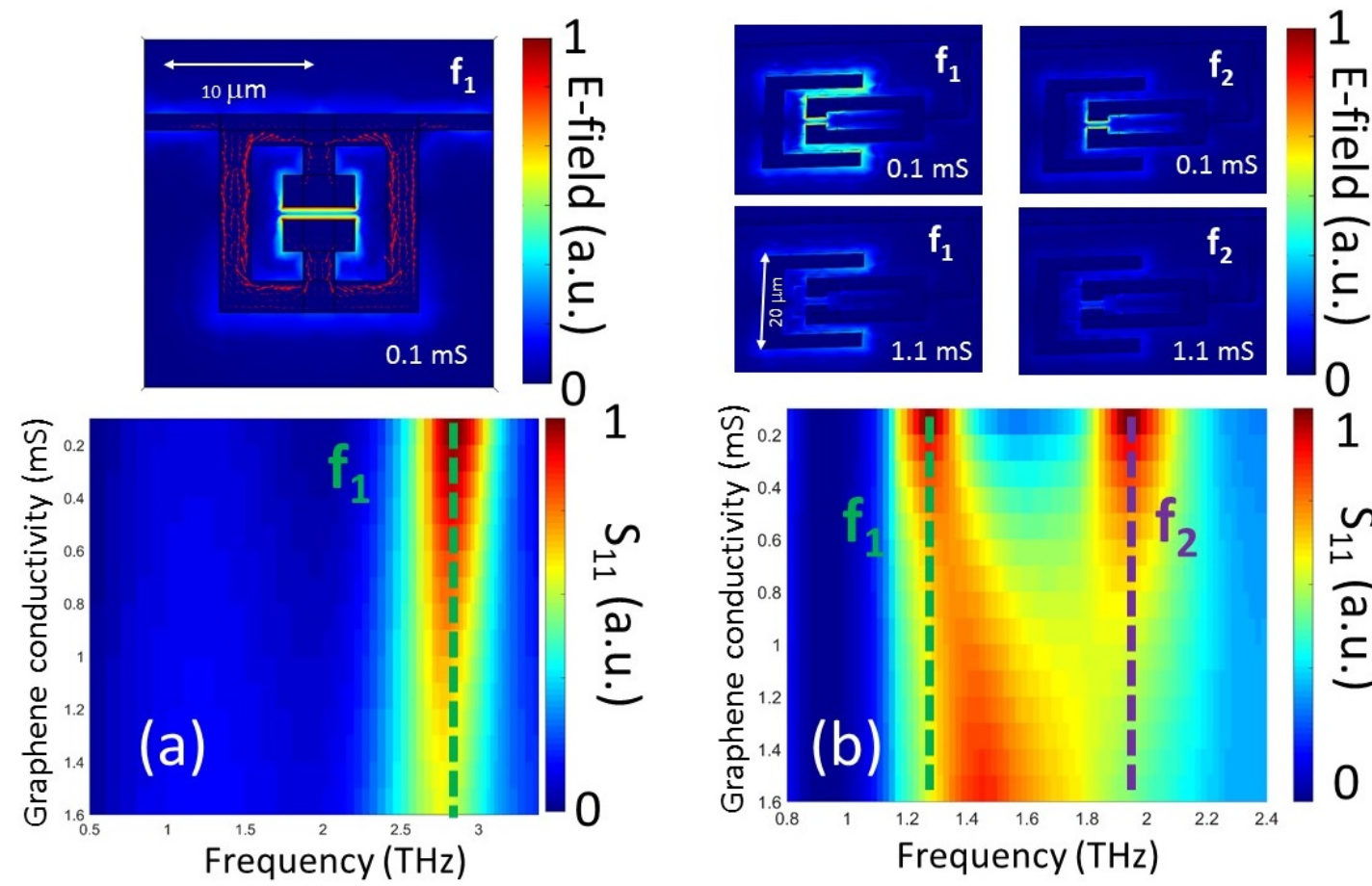

1

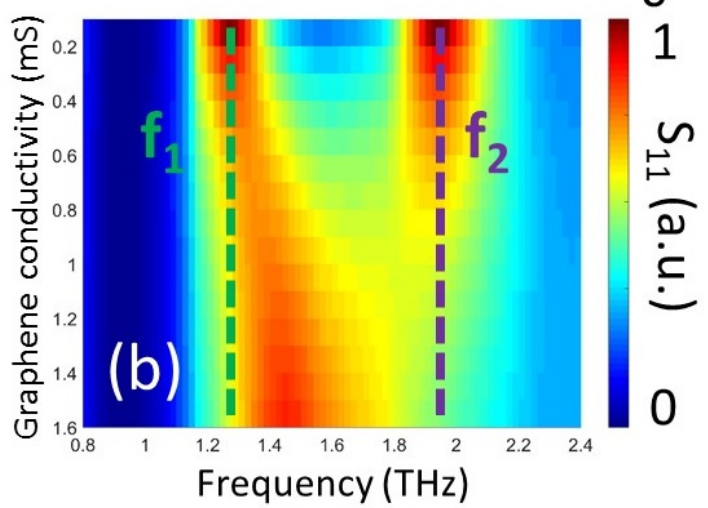

Figure 2. (a) COMSOL simulation of the SRR unit cell, with the E-field at resonance. Below is reported the S11 parameter at different values of graphene conductivity. (b) COMSOL simulation and E-field representation of the EIT unit calculated at frequency f1 and f2 for different values of graphene conductivity EM picture of the SRR arrays for the amplitude modulators. (b) SEM picture of a single resonant unit for the EIT device used for frequency modulation. The graphene patterned region, showed by the dashed area, short the gap in the dark resonators. (c) Schematic of the final device.

\section{EXPERIMENTAL MEASUREMENTS}

\subsection{Broadband THz optical characterization}

In order to validate the simulations and to characterize the optical response of the devices, a time domain spectrometer from Menlosystems, model K15, was used to record the transmission through the devices at various values of the graphene conductivity. The whole optical system was nitrogen purged to reduce water absorption and the time-domain waveform was truncated in order to remove Fabry-Perot multiple resonances from the sample's substrate. In order to experimentally retrieve the graphene conductivity, one graphene area has been processed together with the SRR and EIT arrays but left un-patterned and without any metallic features. The resistance between source and drain pads on this area has been recorded for different back gate voltages by using two 2400 model Keithley source/meter units to retrieve the Dirac point and infer the accessible conductivity range. The THz-TDS and graphene electrical characterizations for the SRR amplitude modulator is reported in reference ${ }^{22}$ and showed remarkable agreement with the simulations. Figure 3(a) shows the normalized THz-TDS transmission through the EIT sample recorded by sweeping the graphene conductivity via the back-gate voltage. The measurements are compatible with the reflection values shown in Fig. 2(b) and present two clear features which have been identified as the bonding and anti-bonding resonances. As expected, by increasing the graphene conductivity/at higher voltages, the dark resonator is dampened, thus shifting the bonding resonance 
towards the single resonator bright resonance. Figure 3(b) presents the resistance between source and drain acquired at different back gate voltages. The Dirac point was found approximately at $100 \mathrm{~V}$ in this configuration where the graphene areas are set at ground potential and the voltage gate was swept between $-100 \mathrm{~V}$ and $100 \mathrm{~V}$. The figure is consistent with the measurements shown in Fig 3(a). Since graphene was left exposed, hysteresis and drift of the position of the Dirac point have been observed, only partly and temporarily restored by thermal annealing. The graphene conductivity range, approximated by the inverse of the measured resistance, is also consistent with the values used in the simulation of Figure 2. The SRR and EIT devices have then been inserted in external cavity for the modulation of quantum cascade lasers and quantum cascade amplifiers (QCA) ${ }^{11}$.

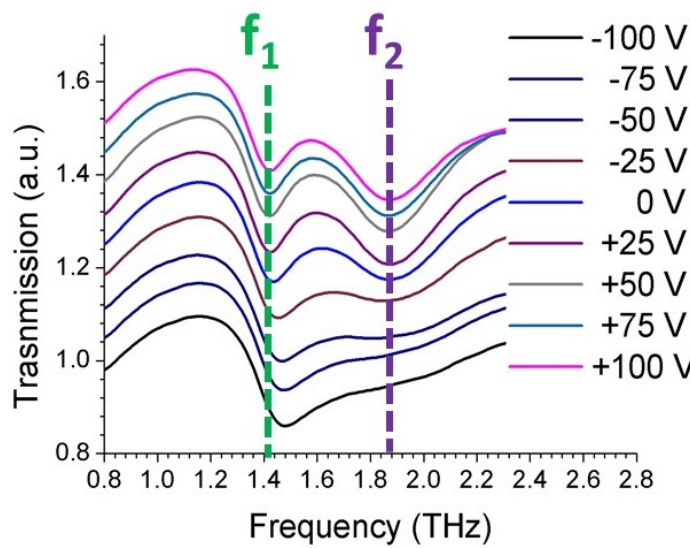

(a)

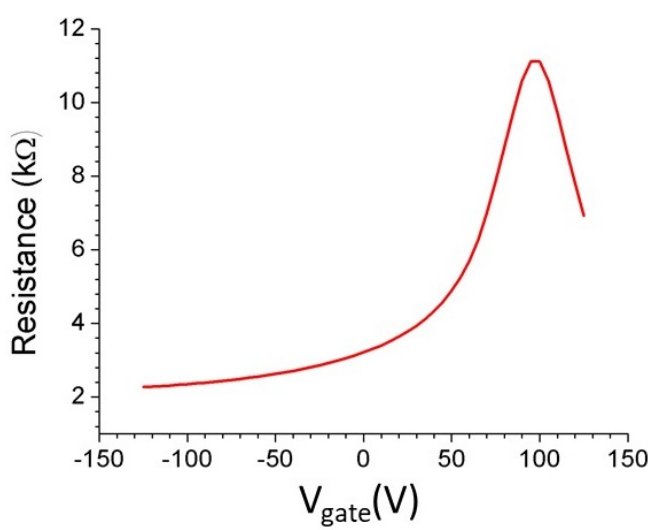

(b)

Figure 3. (a) Measured transmission curve through the EIT device obtained with a THz-TDS spectrometer at different graphene voltages. The two features, $\mathrm{f}_{1}$ and $\mathrm{f}_{2}$, corresponding to the bonding and antibonding frequencies simulated in Figure 2 are clearly distinguishable. The data set is cascaded to improve the visibility. (b) Source-drain resistance of a graphene uniform area fabricated on the same chip of the EIT arrays acquired at different back-gate voltages. The Dirac point was observed at $\sim 100 \mathrm{~V}$.

\subsection{Quantum cascade laser amplitude and frequency modulation}

The various sets of external cavity experiments were carried out with two different QCLs; both bound-to-continuum devices processed with single plasmon waveguide and emitting at $2.9 \mathrm{THz}$ and $2 \mathrm{THz}$. In order to suppress/reduce the reflectivity from the laser facets and to enhance the coupling from the external cavity mirror, hyper-hemispherical antireflection parylene coated silicon lenses were attached to the back facet ${ }^{24}$. An $18.5 \mu \mathrm{m}$ thick parylene coated lens was attached to the back facet of the QCL emitting around $2.9 \mathrm{THz}$ using PMMA, converting the QCL into a quantum cascade amplifier. This operation is critical; a slight misalignment between lens and laser cavity leads to a partial suppression of lasing action. The QCL emitting at $2 \mathrm{THz}$ was only partly suppressed because thicker coatings are more difficult to achieve. For both lasers, the front facet was left uncoated with the output directed into a Fourier-transform infrared spectrometer (Bruker IFS $66 \mathrm{v} / \mathrm{S}$ ). A Si-Ge $4 \mathrm{~K}$ bolometer was used for the acquisition of the spectra, when the QCLs were typically operated with $10 \mathrm{kHz}$ repetition rate, $10 \%$ duty cycle and $\sim 300 \mathrm{~Hz}$ gate frequency. The signal demodulation and acquisition were performed with a lock-in amplifier having the gating frequency as reference and the output of the bolometer as input. Finally, the QCA/QCL devices were mounted onto the cold-finger of a continuous flow liquid helium cryostat with a $\mathrm{THz}$ transparent polyethylene tube used as the outer sheath of the cryostat. Two different external cavity arrangements, schematically illustrated in Figure 4(a)-(b), have been implemented with the reflective metamaterial/graphene feedback elements mounted onto an xyz-stage to complete the external cavity. Figure 4(c) instead reports a schematic picture of the three-mirror model ${ }^{25}$ which can be used to describe the external cavity system, even though for a fully suppressed QCL the standard Lang-Kobayashi ${ }^{26}$ theory cannot be applied. The lasing modes result in first approximation from the overlap between the sub-threshold modes supported by the laser cavity $\mathrm{L}_{\mathrm{c}}$ and the finer comb of the modes supported by the external cavity Lext. The relative weights of the two cavities are given by the respective mirror reflectivity and from the critical coupling of the light reflected back from the external mirror $\mathrm{M}_{\mathrm{ext}}$ into the laser cavity. The dispersive and tunable reflectivity characteristics of the external mirror affects the phase and amplitude of the reflected modes. The set-up arrangements shown in Figure 4(a) was adopted for the QCA in 
combination with the SRR device, as reported in reference ${ }^{22}$. The nominal distance between lens and the SRR mirror was kept fixed to $\sim 1 \mathrm{~cm}$. The EIT devices were implemented with both configurations of Figure 4(a) and 4(b) at a relative distance of $\sim 1 \mathrm{~cm}$ and $\sim 20 \mathrm{~cm}$ (corresponding to the focal position of the second parabolic mirror), respectively. The position of the EIT devices was finely tuned with a nanometer precision motor across these values.

(a)

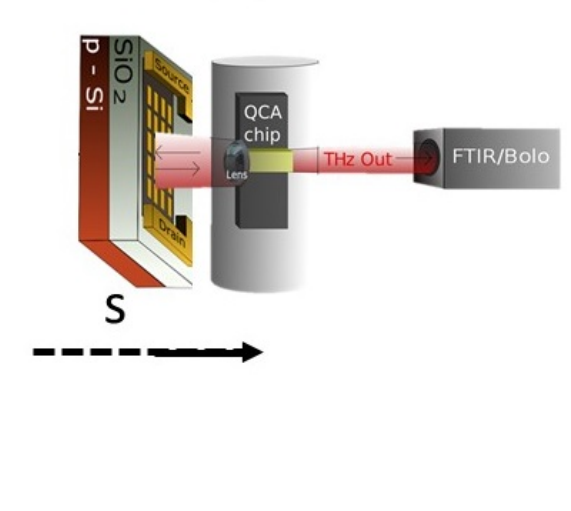

(b)

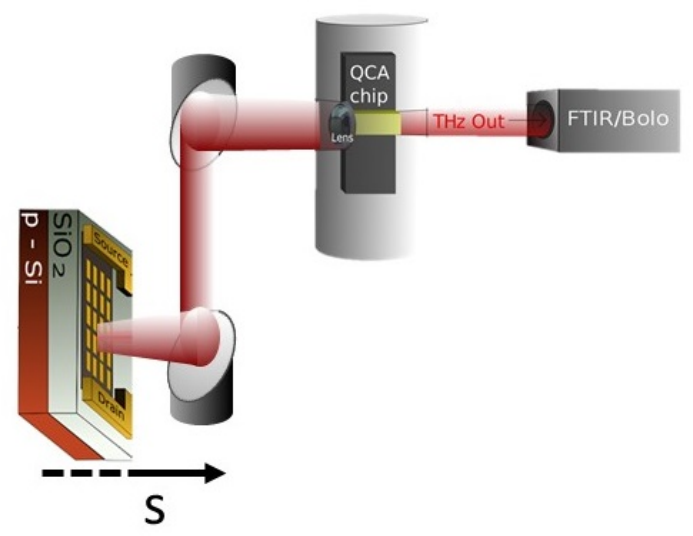

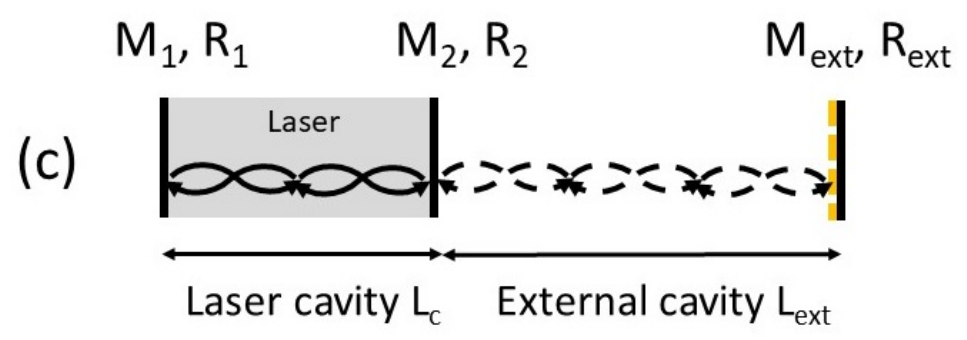

Figure 4. (a) External cavity configuration with the optoelectronic mirror placed as close as possible to the QCA/QCL. (b) In this configuration, two off-axis parabolic mirrors with $7 \mathrm{~cm}$ and $10 \mathrm{~cm}$ focal lengths were used to collimate and focus the emitted radiation onto the modulator array. (c) Simplified schematic of the three-mirror model describing the external cavity arrangement.

In the first set of experiments ${ }^{22}$ with a fully suppressed QCA, a dramatic change in the emitted spectral content in external cavity configuration was reported. The spectra were recorded at different graphene charge carrier concentrations around the Dirac point. Depending on the voltage applied to the SRR mirror and on the relative position of the resonant frequency with respect to the spectral content of the pristine QCL modes, single mode operation was reinforced at the Dirac point for blue or red shift modes. This is exemplified in Figure 5 where single mode operation was achieved at the Dirac point for the lower frequency mode while the second mode appears only when the SRR is biased far from the charge neutrality point. This was attributed to the dynamic change of the mirror reflectivity and also to the different reflected phase seen by the two modes, which although separated by only $50 \mathrm{GHz}$ will experience different feedback and phase change when the graphene conductivity is tuned. This distinctive feature is due to the specific reconfigurable dispersive properties of the SRR optoelectronic device as compared to a standard metallic mirror. Similar experiments have been performed with the EIT device of Figure 3(a) which was mounted on a xyz translational stage for alignment and linear cavity $\mathrm{L}_{\text {ext }}$ scanning in combination with the $2 \mathrm{THz}$ QCL. By linearly scanning the EC length in the configuration of Figure 4(a), it was possible to observe interference fringes, as shown in Figure 6(a). The magnitude and shapes of the fringes are indicative of the feedback strength and of the single/multimode laser emission. A similar EIT device, but lithographically scaled in order to have $\mathrm{f}_{2}$ at approximately $2.85 \mathrm{THz}$ was then used in the configuration of Figure 4 (b) with the QCL emitting around $2.9 \mathrm{THz}$. Due to the inherent difficulty in mounting the coated lens, lasing was strongly reduced but not fully suppressed in this set of measurements. The light-current-voltage (LIV) characteristics of the EC in configuration depicted in Figure 4(b) are shown in Figure 6(b); a significantly larger feedback compared to configuration of Figure 4(a) was recorded in the emitted power. 


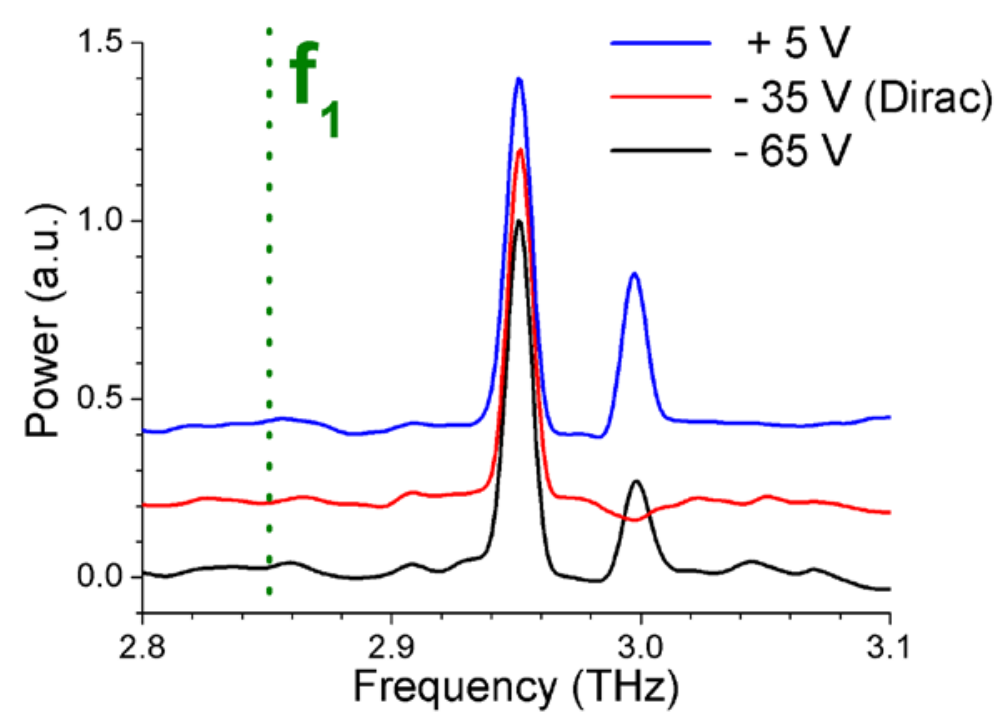

Figure 5. Emitted modes of the external cavity arrangement presented in Figure 4(a) with a SRR mirror at different biasing of the graphene layer. At the Dirac point only the mode closer to the resonant frequency $f_{1}$ of the SRR resonator is present. The spectra are offset in the y-direction for clarity.
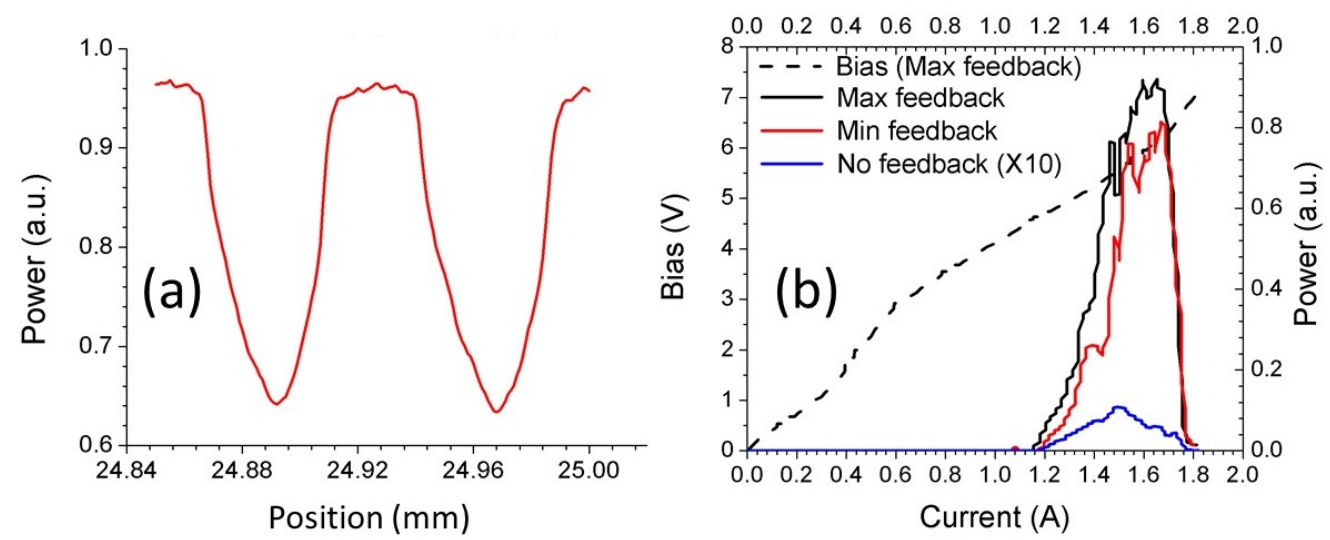

Figure 6. (a) Power recorded in the configuration of Figure 4(a) by scanning the position of the EIT mirror of Fig. 3 (a) with the $2 \mathrm{THz}$ EC-QCL biased at maximum output power. (b) LIV characteristics of the $2.9 \mathrm{THz}$ QCL: with no feedback (blue line); when the EIT mirror resonant at $2.85 \mathrm{THz}$ is in the EC configuration of Figure 4(b) and on the maximum of the interference fringes (black solid line); on the minimum (red solid line).

Compared to the previous configuration, this arrangement allowed a better overlap of the external mirror with the beam spot. An all-electronic control of the spectral emission was performed and the main results are reported in Figure 7 . The measurements acquired at $2.9 \mathrm{THz}$ are reported in Figure 7(a)-(b). The laser was biased with a current of approximately $1.65 \mathrm{~A}$, where the emission was in a range close to mode hopping. As it is shown in Figure 7(a), by performing a scan of the position $s$ with a linearized nanometer precision motor and a mirror instead of the EIT device, a mode hope is observed. This feature can be reproduced with very good approximation by inserting instead of the mirror the EIT device kept at a fixed position and by applying a voltage scan, as shown in Figure 7(b). Similar measurements have been acquired by using the EIT device and EC-QCL centered at $\sim 2$ THz. The configuration chosen was the one in Figure 4(a) and instead of the mirror, the unbiased EIT device was used. As shown in Figure 7(c)-(d), a trend similar to the measurements reported in Figure 7(a)-(b) can be observed, even with a different laser and EIT device. The lower modulation recorded in this last set of measurements is attributed to the reduced feedback achieved by the configuration of Figure 4(a) and to the lower efficiency of the parylene antireflective coating at this frequency. A reproducible, allelectronic control of mode hopping is a novel feature which has never been observed with SRR optoelectronic mirrors 
and it is attributed to the more dispersive nature of the EIT devices. These measurements suggest that the phase change in the modes supported by the external cavity plays a larger role than previously expected, and that a full suppression of the QCL is required in order to enhance the electronic control of EC-QCL emission.
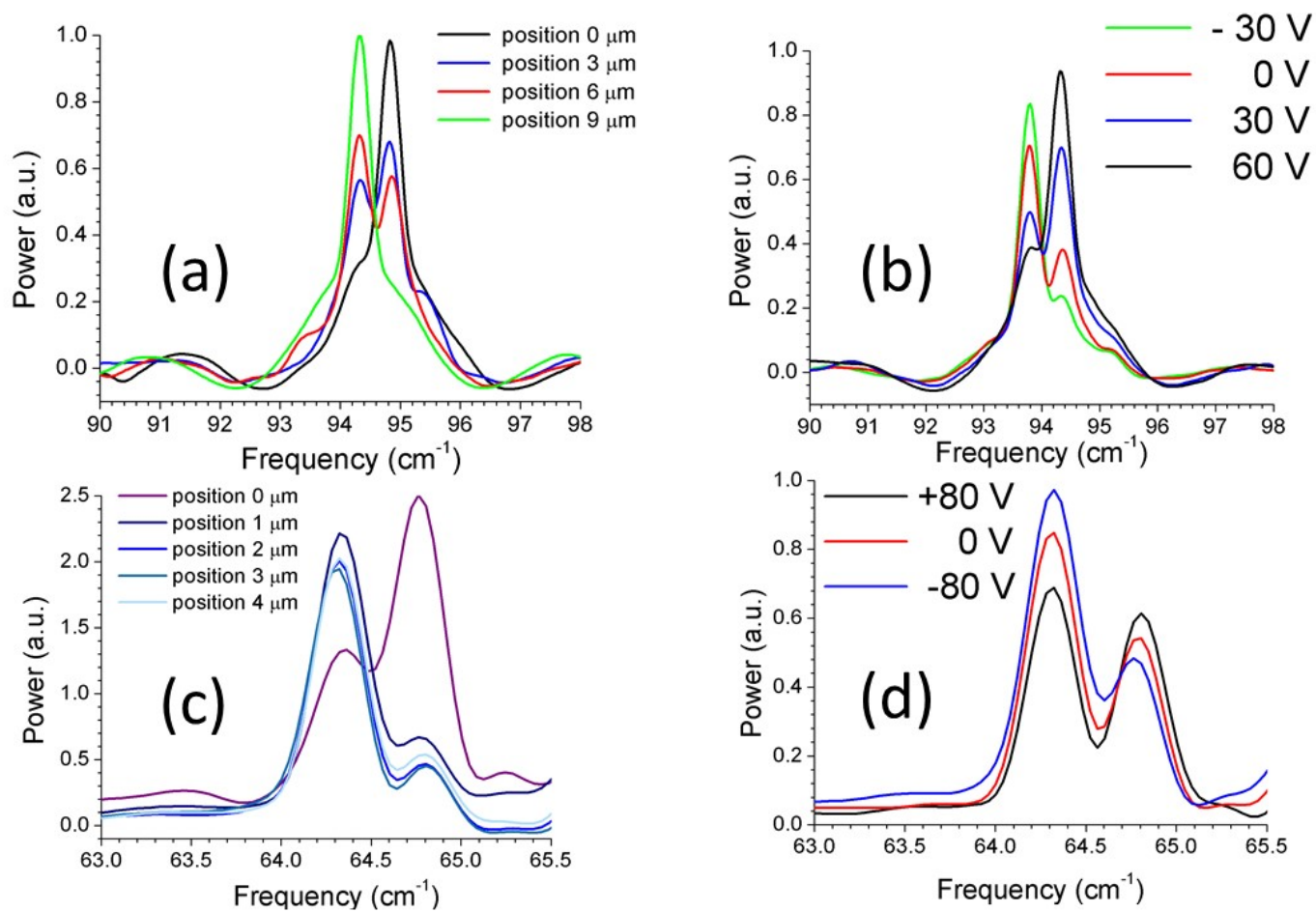

Figure 7. (a)-(b) Spectra for the EC-cavity QCL emitting around $2.9 \mathrm{THz}$ at the current of $1.65 \mathrm{~A}$ acquired (a) by varying the external cavity length of an external mirror (b) by varying the voltage applied to the EIT device kept at a fixed position. The two results show a remarkable agreement. The Dirac point for this EIT device was found to be at 60 V. (c)-(d) Similar measurements performed at 2 THz with the EIT device shown in Fig 3(a). The same trend can be observed even though less pronounced.

\section{CONCLUSION}

In conclusion, amplitude and frequency modulation of QCLs have been reported by using metamaterial SRR and EIT metallic arrays loaded with graphene used as optoelectronic mirrors in external cavity configurations. By varying the graphene carrier concentration around the Dirac point the reflectivity and dispersive characteristics of these devices can be dynamically changed, thus strongly modifying the QCL emission. QCAs or strongly suppressed QCLs have been realized by attaching antireflective coated parylene silicon lenses to the laser facet to enhance the efficiency of the external cavity mirrors. All-electronic, full switching of the EC-QCL emission can be achieved using both devices. Different features in the EC-QCL spectra have been recorded depending on the metamaterial device used. SRR devices, seem to be more efficient in selectively suppressing multimode operation, depending on their relative resonant frequency with respect to the bare QCL emission. EIT devices instead, seem to favor an all-electronic controlled mode hopping. A comparison with standard EC-QCL modulation via external cavity position tuning, reinforce this hypothesis and suggests the use of these fast reconfigurable, versatile integrated devices as a valid alternative in the myriad of fields where external cavity, or frequency selective QCLs find applications in, such as spectroscopy, imaging, and communications.

\section{REFERENCES}

[1] Degl'Innocenti, R., Kindness, S. J., Beere, H. E., Ritchie, D. A., "All-integrated Terahertz modulators," Nanophotonics 7, 127-144 (2018). 
[2] Köhler, R. Tredicucci, A., Beltram, F., Beere, H. E., Linfield, E. H., Davies, A. G., Ritchie, D. A., Iotti, R. C., Rossi, F., "Terahertz semiconductor-heterostructure laser," Nature, 417, 156-159 (2002)

[3] Grischkowsky, D., Keiding, S., Exter, M. V., Fattinger, C., "Far-infrared time-domain spectroscopy with terahertz beams of dielectrics and semiconductors, " J. Opt. Soc. B 7, 2006-2015 (1990).

[4] Degl'Innocenti, R., Xiao, L., Jessop, D. S., Kindness, S. J., Ren, Y., Lin, H., Zeitler, J.A., Alexander-Webber, J. A., Joyce, H. J., Braeuninger-Weimer, P., Hofmann, S., Beere, H. E., Ritchie, D. A., "Fast room temperature detection of terahertz quantum cascade lasers with graphene loaded bow-tie plasmonic antenna arrays," ACS Photonics 3, 1747-1753 (2016).

[5] Degl'Innocenti, R., Xiao, L., Kindness, S. J., Kamboj, V. S., Wei, B., Braeuninger-Weimer, P., Nakanishi, K., Aria, A., Hofmann, S., Beere, H. E., Ritchie, D. A., "Bolometric detection of terahertz quantum cascade lasers with graphene-plasmonic antenna arrays, " J. Phys. D 50(17), 17400 (2017).

[6] Nagatsuma T., Ducournau, G., Renaud, C. C., "Advances in terahertz communications accelerated by photonics," Nat. Photon. 10, 371-379 (2016).

[7] Li, L. H., Chen, L., Freeman, J. R., Salih, M., Dean, P., Davies, A. G., Linfield, E. H., "Multi-Watt high-power THz frequency quantum cascade lasers," Electron. Lett. 53(12), 799-800 (2017).

[8] Richter, H., Pavlov, S. G., Semenov, A. D., Mahler, L., Tredicucci, A., Beere, H. E., Ritchie, D. A., Hübers, H.W., "Submegahertz frequency stabilization of a terahertz quantum cascade laser to a molecular absorption line," Appl. Phys. Lett. 96, 071112 (2010).

[9] Rabanus, D., Graf, U. U., Philipp, M., Ricken, O., Stutzki, J., Vowinkel, B., Wiedner, M. C., Walther, C., Fischer, M., Faist. J., "Phase locking of a 1.5 Terahertz quantum cascade laser and use as a local oscillator in a heterodyne HEB receiver," Opt. Express 17, 1159-1168 (2009).

[10] Ren, Y., Wallis, R., Jessop, D. S., Degl’Innocenti, R., Klimont, A., Beere, H. E., Ritchie, D. A., "Fast terahertz imaging using a quantum cascade amplifier," Appl. Phys. Lett. 107, 11107 (2015).

[11] Ren, Y., Wallis, R., Shah, Y. D., Jessop, D. S., Degl'Innocenti, R., Klimont, A., Kamboj, V. S., Beere, H. E., Ritchie, D. A., "Single mode terahertz quantum cascade amplifier," Appl. Phys. Lett. 105, 141102 (2014).

[12] Rahm, M., Li, J. S., Padilla, W. J., "THz Wave Modulators: A Brief Review on Different Modulation Techniques," J. Infrared Millim. Terahertz Waves, 34, 1-27 (2013).

[13] Padilla, W. J., Taylor, A. J., Highstrete, C., Lee, M., Averitt, R. D., "Dynamical Electric and Magnetic Metamaterial response at Terahertz Frequencies," Phys. Rev. Lett. 96, 107401 (2006)

[14] Liu, P. Q., Luxmoore, I. J., Mikhailov, S. A., Savostianova, N. A., Valmorra, F., Faist, J., Nash, G. R., "Highly tunable hybrid metamaterials employing split-ring resonators strongly coupled to graphene surface plasmons," Nat. Commun. 6, 8969 (2015).

[15] Degl'Innocenti, R., Jessop, D. S., Sol, C. W. O., Xiao, L., Kindness, S. J., Lin, H., Zeitler, J. A., BraeuningerWeimer, P., Hofmann, S., Ren, Y., Kamboj, V. S., Griffiths, J., Beere, H. E., Ritchie, D. A., "Fast modulation of terahertz quantum cascade lasers using graphene loaded plasmonic antennas," ACS Photonics 3, 464-470, (2016).

[16] Jessop, D. S., Kindness, S. J., Xiao, L., Braeuninger-Weimer, P., Lin, H., Ren, Y., Ren, C. X., Hofmann, S., Zeitler, J. A., Beere, H. E., Ritchie, D. A., Degl’Innocenti, R., "Graphene based plasmonic terahertz amplitude modulator operating above $100 \mathrm{MHz}, "$ Appl. Phys. Lett. 108, 171101 (2016).

[17] Braeuninger-Weimer, P., Brennan, B., Pollard, A. J., Hofmann, S., "Understanding and Controlling CuCatalyzed Graphene Nucleation: The Role of Impurities, Roughness, and Oxygen Scavenging," Chem. Mater. 28(24), 8905-8915 (2016).

[18] Zhang, S., Genov, D. A., Wang, Y., Liu, M., Zhang, X., "Plasmon-Induced Transparency in Metamaterials," Phys. Rev. Lett. 101, 047401 (2008).

[19] Qin, Q., Williams, B. S., Kumar, S., Reno, J. L, Hu, Q., "Tuning a terahertz wire laser," Nat. Photon. 3, 732737 (2009).

[20] Kundu, I., Dean, P., Valavanis, A., Chen, L., Li, L., Cunningham, J. E., Linfield, E. H., Davies, A. G., "Quasicontinuous frequency tunable terahertz quantum cascade lasers with coupled cavity and integrated photonic lattice, " Optics Express 25(1), 486-496 (2017).

[21]Wang, F., Nong, H., Fobbe, T., Pistore, V., Houver, S., Merkmann, S., Jukam, N., Amanti, M, Sirtori, C., Moumdji, S., Colombelli, R., Li, L., Linfield, E. H., Davies, A. G., Mangeney, J., Tignon, J., Dhillon. S., "Short Terahertz Pulse Generation from a Dispersion Compensated Modelocked Semiconductor Laser," Laser \& Photonics Reviews 11, 1700013, (2017). 
[22] Kindness, S. J., Jessop, D. S., Wei, B., Wallis, R., Kamboj, V. S., Xiao, L., Ren, Y., Braeuninger-Weimer, P., Aria, A. I., Hofmann, S., Beere, H. E., Ritchie, D. A., Degl'Innocenti, R., "External amplitude and frequency modulation of a terahertz quantum cascade laser using metamaterial/graphene devices," Sci. Rep. 7, 7657 (2017).

[23]Wei, B., Kindness, S. J., Almond, N., Wallis, R., Wu, Y., Ren, Y., Shi, S.-C., Braeuninger-Weimar, P., Hofmann, S., Beere, H. E., Ritchie, D. A., Degl'Innocenti, R., "Amplitude stabilization and active control of a terahertz quantum cascade laser with a graphene loaded split-ring-resonator array," Appl. Phys. Lett. 112, 201102 (2018).

[24] Degl'Innocenti, R., Shah, Y. D., Jessop, D. S., Ren, Y., Mitrofanov, O., Beere, H. E., Ritchie, D. A., "Hollow metallic waveguides integrated with terahertz quantum cascade lasers," Optics Express 22(20), 24439-24449 (2014).

[25] Taimre, T., Bertling, K., Leng Lim, Y., Dean, P., Indjin, D., Rakic, D. A., "Methodology for materials analysis using swept-frequency feedback interferometry with terahertz frequency quantum cascade lasers," Optics Express 22(15), 18633-18647 (2014).

[26] Lang, R., Kobayashi, K., "External optical feedback effects on semiconductor injection laser properties," IEEE J. Quant. Electron. 16, 347-355 (1980). 